\title{
LOCALLY INTERPOLATING SUBDIVISION SURFACES SUPPORTING FREE-FORM 2D DEFORMATIONS
}

\author{
J. Claes ${ }^{1}$, F. van Reeth ${ }^{2}$ and M. Ramaekers ${ }^{2}$ \\ ${ }^{1}$ University of the Balearic Islands, Spain; ${ }^{2}$ Limburg University Centre, Belgium
}

Key words: free-form deformation, 2D computer animation, subdivision surfaces, Loop subdivision, texture mapping

Abstract: This paper addresses the application of free-form deformations to arbitrarily shaped 2D textured objects, solving specific problems. Based on subdivision surfaces applied in 2D, our method successfully combines the following features: fluid good-looking movement, both general global and precise local control and explicit discontinuities. Moreover we implemented an extension to the approximating subdivision scheme, providing local interpolation and accurate border control.

\section{INTRODUCTION}

This paper describes a free-form deformation scheme dealing with $2 \mathrm{D}$ animated objects. As animations are mostly shown as moving 2D images, it often suffices to decide about the movements in two dimensions only to create convincible animations. This does not work out properly when physically correct movements are needed, but is very suitable to informally deliver creative ideas to a viewer.

The following requirements showed up for free-form deformations suited to accomplish this goal:

- Allow fluid movement, not only at the border, but also at the interior of the animated object, making sure the texture parameterisation of the surface is deformed in a smooth, natural looking way. 
- Both global control - needing limited user interaction - and fine local control near specified joints should be integrated into one consistent interface paradigm.

- Allow specific discontinuities; this can be a hole inside the animated object or limbs sticking out from it. E.g. although two feet of a character can be situated close together, usually they should be animated and deformed independently. Moreover, they even might overlap.

In order to cope with all this, we closely examined existing free-form deformation schemes, but unfortunately none of them combined all desired requirements. Therefore we opted to investigate the application of the extensions of the subdivision scheme described in [VanRe01].

The rest of this work is organised as follows. Section 2 describes freeform deformations and explains how they will be used in our application. Section 3 deals with subdivision surfaces with local interpolation. In section 4 the details of our implementation are elucidated, while the next section describes our ongoing future work.

\section{FREE-FORM DEFORMATION (FFD) IN 2D}

\subsection{Existing FFD schemes}

Sederberg [Seder86] and Barr [Barr84] were about the firsts to point out possibilities, advantages and implementation schemes of deformations, and more in particular of free-form deformations (FFDs). Many followed this trail, improving and extending their usability for different tasks and requirements.

Sederberg put a 3D B-spline lattice around a selected object, then modified the positions of the vertices of the control lattice, and finally applied that deformation to the object. Coquillart combined Sederberg's lattices to allow more complicated deformations [Coqui90]. In a follow-up paper, she also decoupled the lattice from the object to allow animating the lattice separately or to move the object through a deformed space [Coqui91].

Different representations of the deformation tool were investigated:

- a surrounding control lattice [Seder86],

- combining multiple lattices [Coqui90],

- a lattice build up from subdivision volumes [MacCr96],

- some controlling curves or based on an axis [Barr84],

- control surfaces [Feng96] or

- a scattered set of points [Mocc097].

The type of tool used for the deformation strongly determines which type of deformations are feasible and how easy the user can control them. Each 
tool can be adequate in its own right, depending on the needs in the specific application.

Most of the work in FFDs is concentrating on 3D deformations, considering 2D deformations as a simplification: just leave out one dimension. Hereby ignoring that when you restrict yourself to $2 \mathrm{D}$ deformations, additional goals can be achieved as explained in the introduction (see section 1). One of the people specifically tackling 2D deformations was Sederberg in his Siggraph'93 paper [Seder93], where he describes a method to interpolate between 2 deformed 2D objects. Each object is represented by a polygon. The paper restricts itself to the behaviour of the border, giving no clue about how the interior of the polygons should be deformed.

\subsection{Deforming parameterisation and local control}

In [Inter97] arguments are given to show the significance of texture mapping for conveying 3D shape, even for non-deformable objects. Moreover, when we only have a flat 2D deformable object that pretends to represent a 3D shape, precise control of the texture mapping becomes extremely important in order to deform in a convincible way.

Zonenschein et al. [Zonen98] studied the texturing of deformable implicit surfaces, indicating texture artefacts ("ghosting") when the objects are deformed. They needed to blend colours and transformations to get a plausible result. We opt for a more exact control of the texture, so we try to avoid blending.

The FFD schemes mentioned in section 2.1 do not specifically take care of the parameterisation (texturing) of the surface: they only concentrate on the general shape. Furthermore, with most of these FFD schemes, local control is not so easy. Local control implicates a denser mesh, but usually this is only possible if the complete mesh is subdivided, which obliges the user to control a huge set of points. Only [Mocco97] and [MacCr96] allow local control, so their approaches needed to be studied closer in view of our application.

We considered the approach of [Mocc097], who organises scattered control points into a Delauney triangulation. Their mesh is not explicitly visible to the user, which has the advantage that the user doesn't need to spend time to create the connections, but has the disadvantage that the user can't make different connections when needed, for example to mimic certain physical connections. As the main goal in [Mocco97] is deforming hands represented by many control points that are positioned relatively close together, a Delauney triangulation forms the most adequate connectivity. When attempting to apply this approach for $2 \mathrm{D}$ animation purposes however, 
with only a limited number of control points, the possibility to create own connections - including explicit discontinuities - turned out to be a necessity. Nevertheless, [Mocco97]'s idea to start out with a Delauney triangulation is also useful in our approach, where we extend the idea with the possibility to re-edit the generated mesh. Unfortunately their scheme to calculate the coordinates in the mesh will not be applicable anymore, as it strongly depends on the Voronoi diagrams defined by these triangulations. Furthermore the convex hull property prohibits having the type of discontinuities we need.

MacCracken and Joy's solution to FFDs [MacCr96] is based on subdivision volumes created by 3D lattices of arbitrary topology. We liked their idea to use subdivision, as it is the only FFD approach facilitating arbitrary topologies. Nevertheless - although in theory there is a lot of freedom in manipulating the deformation - their set-up is rather hard to establish and control by a user. Also, their way of subdividing space makes calculating the co-ordinates of a point referring to the deforming mesh less straightforward. In our approach, instead of their 3D subdivision volumes, we apply subdivision surfaces, augmented with adequate control tools.

The system we propose has specific advantages and features when comparing it to the previously described techniques. None of the techniques combines all of these features into one concise interface. The main differences are:

a) We allow both general global local in areas of less interest and simultaneously precise local control where needed. This combined type of control is also possible in [MacCr96], but their 3D lattices are hard to handle and to position precisely, and furthermore they don't allow for local interpolations. [Mocc097] also allows some combination of local and global control, but does not provide discontinuities.

b) None of the FFD techniques described in section 2.1 explicitly cares for what happens to the object outside of the border. Objects are just embedded in a larger space. Everything that would happen with the FFD transformation outside of the border will just be ignored. In our approach however, we want to allow for discontinuities. If the transformation would extend too far outside the border, the effect of an FFD applied to one part will result in an overlap with neighbouring parts of the object. This overlapping complicates making sure the animation of one part does not influence a neighbouring part, for example in the case of two legs. Therefore we provide very precise border control.

c) Most FFD approaches can easily deform an object as a whole, but have problems handling the interior just as easy. The interior is deformed as to minimise distortions, but this can't be guided as fluently as desired by an animator. We solve this by allowing for interpolating points, not only at the border but also at the interior. 


\section{LOCALLY INTERPOLATING SUBDIVISION SURFACES}

\subsection{Recursive subdivision schemes}

Recursive subdivision schemes have been used to define curves (in 2D or in 3D), surfaces (usually in 3D) and volumes (in 3D) [MacCr96]. Such a scheme starts with a set of control points, and in each subsequent subdivision step, in-between points are introduced and at the same time the points are averaged by their neighbours. Depending on how adequate the averaging scheme is, this process will eventually converge to a smooth curve, surface or volume. It will result in a curve if the points are connected in one linked list (like a polygon), in a surface if the points are connected like a polyhedron and in a volume with points connected in a lattice. A good introduction to recursive subdivision schemes can be found at [Joy96], while [Zorin00] provides an in-depth overview of the state of the art.

\subsection{Using subdivision surfaces for FFD}

In this paper, we based our FFD scheme on subdivision surfaces, as such a surface can both represent the border and the interior of a $2 \mathrm{D}$ object. A subdivision scheme is said to be uniform if the same scheme is applied unchanged to every point. The scheme is stationary if the same rules are used for all subsequent subdivisions. As interesting mathematical and practical properties require the scheme to be both stationary and uniform, people only avoid them if they want to achieve exceptional goals. One of these goals can be coping with boundary conditions, because the ordinary rules for the interior do not work at the border. As we want to describe a 2D surface that does not cover the entire plane, we necessarily need to have surfaces with a border. Luckily the standard rules for borders keep the properties of the otherwise fully uniform Loop scheme intact.

In [Catmu78] a subdivision scheme is described, that became known as Catmull-Clark subdivision surfaces. Their scheme is both uniform and stationary and has been studied extensively. It turned out to be very adequate for practical use and lends itself to extensions like sharp edges, which were used in real productions, like the animated short "Gerry's Game".

The Catmull-Clark scheme is an approximating scheme: the limit surface smoothly approximates the mesh of initial control points, and normally will not interpolate them. On the other hand, also completely interpolating schemes exist, for example the Butterfly scheme, introduced in [Doo78] and later extended, amongst others by [Dyn90] and [Zorin96]. Interpolating schemes have the advantage that all initial points will be interpolated 
exactly, which gives good control about their position and makes them useful for fitting a set of digitised points. In practice it turned out that they are not so well suited for interactive editing, as they need a larger support area and unwanted bulges and folds are difficult to control. Moreover for the described approximating schemes it can be guaranteed that the bounding box set out by the control points will contain the entire generated surface, and this property is maintained recursively throughout the subdivision process.

[Halst93] describes a way to convert the approximating Catmull-Clark scheme into a scheme interpolating a set of given points, using a global optimisation technique. They calculated a new mesh of control points for which the limit surface will interpolate the original points. In order to make the surface sufficiently fair, not only the initial points but also the points of the first and second subdivision needed to be moved. Hence, the scheme of [Halst93] gets similar drawbacks for interactive editing, as other fully interpolating schemes.

Apart from the Catmull-Clark subdivision surfaces, another approximating scheme got quite popular: Loop's scheme [Loop87]. While Catmull-Clark subdivision is based on dividing the subsequent meshes into quadrilaterals, Loop is purely working with triangles. When looking at our application - deformations - the use of triangles has some advantages over quadrilaterals. For instance, a point inside a triangle can be consistently expressed by a co-ordinate system set up by the 3 points of the triangle, while a quadrilateral needs to be subdivided into 2 triangles to get the same consistency.

Because of the above-mentioned reasons - the ease of editing control and the appropriateness for our application - we chose to work with an approximating scheme based on triangles: Loop's subdivision surfaces.

\subsection{Local interpolation, normal and tension control}

As we wanted to have better control over the surfaces, we opted to extend Loop's scheme with interpolation on selected points, without losing advantages such as fairness and the convex hull property. [Levin00] also describes a method to make a scheme locally interpolating, but he does this by applying other weights around the points to be interpolated, hereby turning a uniform scheme into a non-uniform one. This causes an implementation to lose many of the benefits of the original scheme, like not having to deal with local exceptions and maintaining the convex hull property.

First, let's have a look at the formulas of Loop's subdivision scheme. Loop surfaces are built up starting from a mesh of triangles. During each subdivision step, first the mesh is split, introducing a new point at the centre 
of every edge; these points get interconnected to form a new mesh, having each triangle divided into four new triangles. Then all points are averaged in order to become a smooth surface. This process is executed recursively, resulting in a fine subdivided mesh of small triangles, in the limit forming a surface. The moved points at the centre of an edge are called edge points, while the points of the existing mesh are called vertex points. The rule for adding a new edge point $E$ (on the edge between $V_{1}$ and $V_{2}$ and with $Q_{1}$ and $\mathrm{Q}_{2}$ as immediate neighbours) in the interior of the mesh (see figure 1 ) is:

$$
V_{0}^{\prime}=\sum \beta Q_{i}+(1-k \beta) V_{0} \quad \text { with } \quad \beta=\frac{1}{k}\left(\frac{5}{8}-\left(\frac{3}{8}+\frac{1}{4} \cos \frac{2 \pi}{k}\right)^{2}\right)
$$

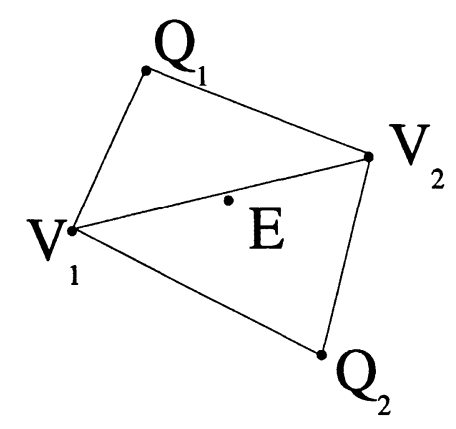

Figure 1. Situation around an interior edge

And the rule for averaging an interior point $\mathrm{V}_{0}$ (surrounded by $\mathrm{k}$ vertices $\mathrm{Q}_{1} \ldots \mathrm{Q}_{\mathrm{k}}$ ) is the following (see figure 2):

$$
E=\frac{3}{8}\left(V_{1}+V_{2}\right)+\frac{1}{8}\left(Q_{1}+Q_{2}\right)
$$




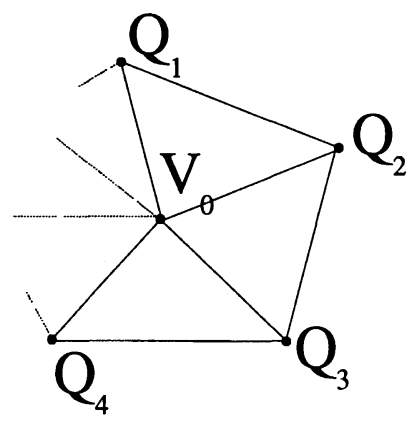

Figure 2. Situation around an interior vertex

The formulas for new edge and vertex points at the border, are simply (see figure 3):

$$
V_{0}^{\prime}=\frac{3}{4} V_{0}+\frac{1}{8}\left(V_{1}+V_{2}\right) \text { and } E=\frac{1}{2}\left(V_{1}+V_{2}\right)
$$

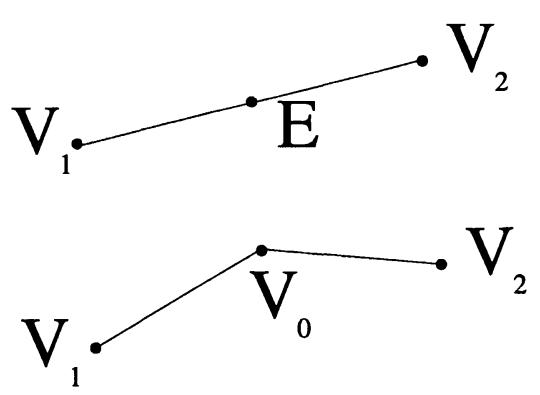

Figure 3. Situation around a border edge and a border vertex

From these formulas it is clear that the border of the Loop surfaces is just a subdivision curve, without influence from interior points. This makes the technique described in [VanRe01] dealing with locally interpolating subdivision curves, valuable for our FFD implementation. Local interpolation is accomplished by extending the control polygon of the curve with ghost points on a line throughout the point to be interpolated. The orientation of this line controls the tangent (thus the normal) at the interpolated point, while the distance between the ghost points affects the tension. Hence, besides local interpolation, the described techniques also provide normal and tension control, without having to revert to a non- 
uniform scheme. See figure 4 for an example. Extra details can be found in [VanRe01].
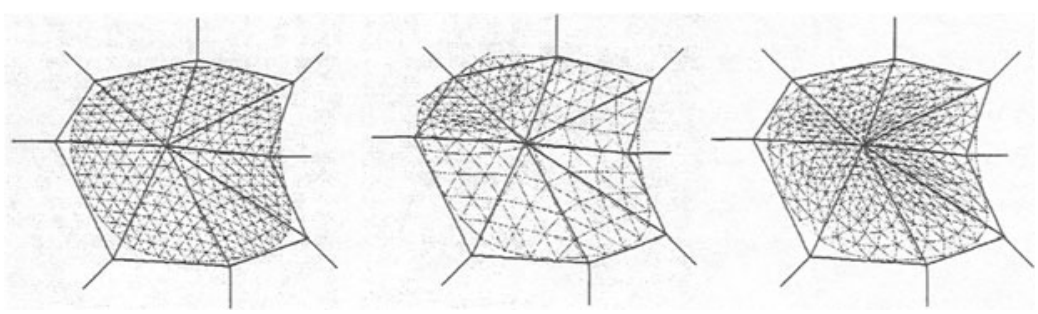

Figure 4. A subdivision mesh, at the left without interpolation, at the centre interpolating a border vertex, and at the right interpolating an interior point (note that for clarity also the normals at the border edges are shown).

The technique described in [VanRe01] can furthermore be extended to the interior of the Loop surfaces. If we want the surface to interpolate an interior control point, the surrounding ghost points should be set up in such a way that their average equals the point to be interpolated. Equation 1 makes sure that if a vertex $\mathrm{V}_{0}$ is on the given location before the subdivision, it will stay on that same position after the subdivision step. More particularly, the condition to have $V_{0}$ being the average of the surrounding vertices can be written as:

$$
V_{0}=\frac{1}{k} \sum Q_{i}
$$

and substituting this into eq.1 leads to $V_{0}^{\prime}=\beta k \frac{1}{k} \sum Q_{i}+(1-k \beta) V_{0}$ or $V_{0}^{\prime}=V_{0}$

And thanks to equation 2, the edge points around $V_{0}$ will again have $V_{0}$ as their average.

$$
\begin{aligned}
& \frac{1}{k} \sum E_{i}=\frac{1}{k} \sum\left(\frac{3}{8}\left(V_{0}+Q_{i}\right)+\frac{1}{8}\left(Q_{i+1}+Q_{i-1}\right)\right) \\
& \text { or } \frac{1}{k} \sum E_{i}=\frac{1}{k} \sum \frac{3}{8} V_{0}+\frac{1}{k} \sum \frac{5}{8} Q_{i}
\end{aligned}
$$




$$
\text { or } \frac{1}{k} \sum E_{i}=\frac{3}{8} V_{0}+\frac{5}{8}\left(\frac{1}{k} \sum Q_{i}\right)
$$

This makes the average of the new edge points equal to $V_{0}$ provided that the $\mathrm{Q}_{\mathrm{i}}$ also have $\mathrm{V}_{0}$ as their average. Applying this knowledge in a recursive way will ensure that for all subsequent subdivision steps, the interior vertex $\mathrm{V}_{0}$ will constantly stay on the same position.

Figure 4 is an example of a mesh with the two sorts of interpolation.

\section{IMPLEMENTATION}

In our basic approach, we start out with a 2D object to be deformed. The object is represented by a $2 \mathrm{D}$ image and can have an arbitrary topology, as it can have holes or limbs sticking out. On this image, the user draws a net of control points. The control points are put both at the interior and near the border. At most places, just an approximating control mesh suffices, but wherever the user needs more control, she can choose to insert an interpolating point (see section 3.3). The user can create some kind of skeleton using this mesh, but this is not a necessity.

Furthermore, special care is taken at the border near places where separate parts that stick out come close together, for example between the upper lip and the nose. In more traditional FFD approaches, at those places the control mesh would be interconnected, forming something like a convex hull. We will draw the discontinuities explicitly, by having the border being continued between them.

When the user finishes setting up the initial mesh, this mesh is frozen to the object, analogous to other FFD approaches. Internally in our program, the mesh will be converted into the triangles belonging to some levels of subdivision. At the corner of each triangle, texture co-ordinates will be generated, mapping the undeformed initial 2D image to this geometry.

In the next stage - also a typical step in FFD - the user can start moving points of the control mesh or even animate them. In the program, the mesh will be subdivided again, the texture co-ordinates belonging to the initial position will be applied and everything will be redrawn, resulting in a deformed object. Typical for our approach, is that apart from moving control points, the user can also manipulate the tension and the normal, giving rise to appealing effects that are hard to establish with other methods. Figure 5 shows a typical example. 

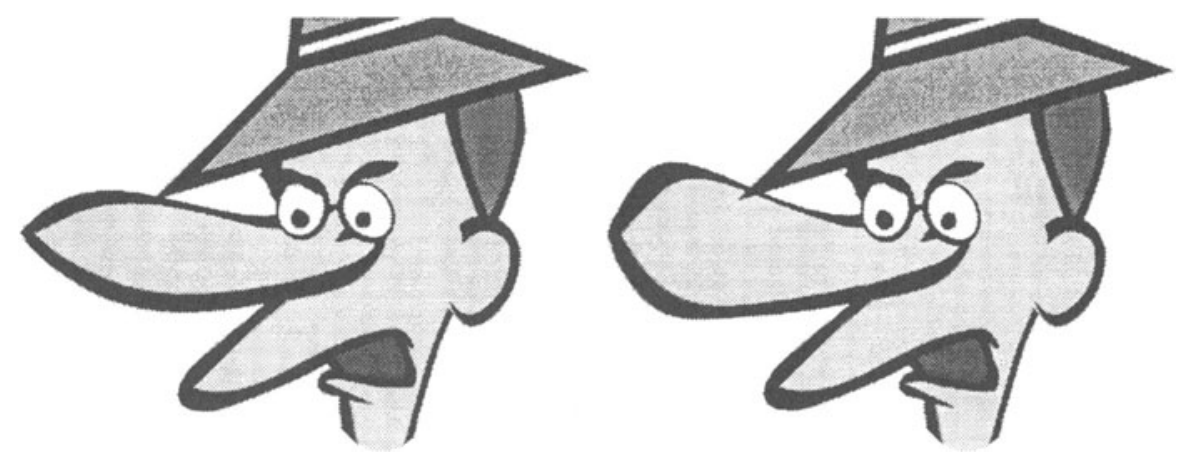

Figure 5. An example of only changing the tension in the border point at the tip of the nose.

An additional advantage of working with the approximating Loop subdivision scheme, is that we can set up a tree of bounding boxes, where each subsequent subdivision level is contained into a bounding box set up by the control points of that level. This enables a quick search for where a point resides into the generated mesh.

Figure 6 refers to a very expressive animation that was created with just a small amount of user input. The animation gives a lot of 3D feeling, while all manipulations are kept strictly $2 \mathrm{D}$.
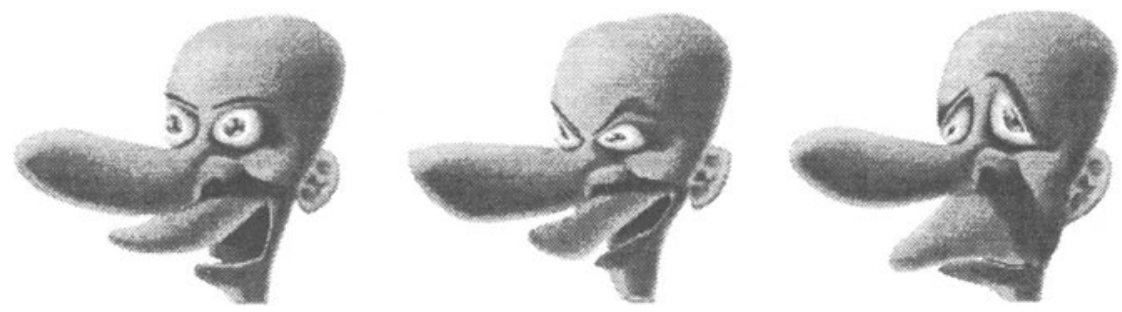

Figure 6. Some frames from an animation created by our system. Interpolating points are used around the eyes, to provide better local control. Between the upper lip and the nose there is an explicit discontinuity to prevent that moving the lip would have undesired effects on the nose.

\section{CONCLUSIONS AND FUTURE WORK}

In this paper we described a method for deforming 2D images, based on locally interpolating subdivision surfaces with normal and tension control. Our method enables a very smooth movement, explicit discontinuities and 
both global and local control. None of the other FFD approaches, described in section 2.1 is able to combine all these features into one uniform concept.

In our ongoing future work, we are investigating ways to incorporate higher level editing of the mesh, for example multi-resolution editing. Also we want to have a closer look at combining our methods with physically based modelling techniques and constraint based systems.

Also we are thinking about extending our approach to 3D with keeping in mind the requirements that are also important for $2 \mathrm{D}$ deformations. Another track is instead of deforming objects, deforming the space through which the object moves - similar to the ideas presented in [Coqui91].

\section{ACKNOWLEDGEMENTS}

The authors are very pleased to acknowledge that this work has been partially funded by the European TMR (Training and Mobility of Researchers) project PAVR (Platform for Animation and Virtual Reality), the European Fund for Regional Development and The Flemish Government. Also the creative input from Luis Gutierrez and Joan Cabot is highly appreciated.

\section{REFERENCES}

[Barr84] A. Barr, "Global and Local Deformation of Solid Primitives", Computer Graphics, Vol.18, No.3 (Proc. Siggraph'84), pp. 21-30, 1984.

[Catmu78] E. Catmull, J. Clark "Recursively Generated B-spline Surfaces on Arbitrary Topological Meshes", Computer-Aided Design 10 (Sept. 1978), pp.350-355.

[Coqui90] S. Coquillart, "Extended Free-Form Deformation: A Sculpturing Tool for 3D Geometric Modeling”, Siggraph 90, pp.187-196, August 1990.

[Coqui91] S. Coquillart, "Animated Free-Form Deformation: An Interactive Animation Technique”, Siggraph 91, pp. 23-26, July 1991.

[DeRose98] T. DeRose, M. Kass, T. Truong, "Subdivision Surfaces in Character Animation", SIGGRAPH 98 Conference Proceedings (July 1998), pp.85-94.

[Doo78] D. Doo, M. Sabin "Behaviour Of Recursive Division Surfaces Near Extraordinary Points”, Computer-Aided Design 10 (Sept. 1978), pp.356-360.

[Dyn90] N. Dyn, J. A. Gregory, D. Levin, “A Butterfly Subdivision Scheme for Surface Interpolation with Tension Control." ACM Transactions on Graphics. Vol. 9, No. 2 (April 1990), pp. 160-169.

[Feng96] J. Feng, L. Ma, Q. Peng, “A New Free-Form Deformation Through the Control of Parametric Surfaces”, Computers \& Graphics, Vol.20, No.4, pp. 531-539, 1996.

[Halst93] M. Halstead, M. Kass, T. DeRose, "Efficient, Fair Interpolation using CatmullClark Surfaces", Proceedings of Siggraph 93, pp. 35-44, 1993.

[Inter97] V. Interrante, H. Fuchs, S. Pizer, "Conveying the 3D Shape of Smoothly Curving Transparent Surfaces via Texture", IEEE Transactions on Visualization and Computer Graphics, vol. 3, no. 2, pp. 98-117, April-June 1997. 
[Joy96] K. Joy, “On-Line Geometric Modeling Notes”, webpages available at http://muldoon.cipic.ucdavis.edu/CAGDNotes/, 1996.

[Levin00] A. Levin, "Surface Design using Locally Interpolating Subdivision Schemes", Journal of Approximation Theory, Vol. 104, No. 1, pp. 98-120, May 2000.

[Loop87] C. Loop, “Smooth Subdivision Surfaces Based on Triangles”, Master's thesis, University of Utah, Department of Mathematics, 1987.

[MacCr96] R. MacCracken, K. Joy, "Free-Form Deformations with Lattices of Arbitrary Topology”, Siggraph'96, pp.181-188, August 1996.

[Mocc097] L. Moccozet, N. Magnenat Thalmann, "Dirichlet Free-Form Deformations and their Applications to Hand Simulation", Proc. Computer Animation, IEEE Computer Society, pp. 93-102, 1997.

[Seder86] T. Sederberg, S. Parry, "Free-Form Deformation of Solid Geometric Models", Siggraph 86, pp.151-160, 1986

[Seder93] T. Sederberg, P. Gao, G. Wang, H. Mu, “2-D Shape Blending: an Intrinsic Solution to the Vertex Path Problem", Siggraph 93, pp.15-18

[VanRe01] F. Van Reeth, J. Claes, "Interpolatory Uniform Subdivision Curves with Normal Interpolation and Tension Control, Tenerating B-splines of Any Degree", to be submitted to The Visual Computer.

[Zonen98] R. Zonenschein, J. Gomes, L. Velho, L. H. de Figueredo, M. Tigges, B. Wyvill, "Texturing Composite Deformable Implicit Objects", Proceedings of the XI International Symposium on Computer Graphics, Image Processing and Vision, pp. 346-353, Rio de Janeiro, October 1998.

[Zorin96] D. Zorin, P. Schröder, W. Sweldens, "Interpolating Subdivision for Meshes with Arbitrary Topology", Tech. Rep. CS-TR-96-06, Caltech, Department of Computer Science, 1996.

[Zorin00] D. Zorin, P. Schröder, A. Levin, L. Kobbelt, W. Sweldens, T. DeRose, "Subdivision for Modeling and Animation", course SIGGRAPH 2000. 